\title{
Repeated vaginal administration of trimeric HIV-1 clade C gp140 induces serum and mucosal antibody responses
}

\author{
MP Cranage ${ }^{1}$, CA Fraser $^{1}$, Z Stevens $^{1}$, J Huting $^{1}$, M Chang $^{1,2}$, SA Jeffs $^{3}$, MS Seaman $^{4}$, A Cope $^{1}$, T Cole $^{1}$ \\ and RJ Shattock ${ }^{1}$
}

\begin{abstract}
Vaccine-mediated prevention of primary infection with human immunodeficiency virus (HIV) may require the sustained production of antibody at mucosal portals of entry. Here, we describe a novel approach of repeated mucosal immunization by delivering an HIV-1 envelope glycoprotein (gp) in a gel formulated for intravaginal delivery. Rabbits were immunized over one to three 19-day cycles of intravaginal dosing with soluble recombinant trimeric HIV-1 clade C gp140 administered in Carbopol gel. The formulation was well tolerated. A single immunization cycle induced immunoglobulin G (IgG) antibody detected in the serum and female genital tract, and titers were boosted on further immunization. Vaccine-induced serum antibodies neutralized the infectivity of a pseudovirus carrying a heterologous clade $\mathrm{C}$ envelope. Our data prove the concept that repeated exposure of the female genital tract to HIV envelope can induce mucosally detectable antibody.
\end{abstract}

\section{INTRODUCTION}

Developing a vaccine to protect against infection with human immunodeficiency virus (HIV) is complicated by a series of critical issues, not least of which is the problem of how to stimulate protective immunity at mucosal portals of virus entry. In sub-Saharan Africa, heterosexual transmission remains the driving force of the epidemic with women disproportionately infected, particularly those in the 15-24 year age group. ${ }^{1}$ The exact mechanisms whereby HIV infects across a mucosal barrier are still under intense investigation (reviewed by Shattock and Moore ${ }^{2}$ ); however, studies with simian immunodeficiency virus (SIV) show that once the virus has crossed the vaginal epithelium there is rapid dissemination, although systemic replication may be somewhat delayed. ${ }^{3}$ On account of this rapid infection dynamic, and because HIV targets the immune system directly and integrates proviral DNA into the genome of target cells, a fully effective HIV vaccine, rather than one that is limited to reducing virus load, will likely completely prevent disseminated infection by stimulating immune effector function actually in the mucosal environment. At present, it is uncertain how to achieve this. Certainly, antibodies have the potential to be effective: intravenous infusion of monoclonal neutralizing antibodies has been shown to protect macaques against vaginal challenge with $\operatorname{SIV}^{4,5}$ however, vaccine-induced immunity is believed to rely mainly on recall of immunological memory, and the conditions required for the sustained production of antibody at the mucosal surface are yet to be established.

The human female genital tract is a site of both inductive and effector immune functions, which are strongly associated with the phases of the menstrual cycle. ${ }^{6}$ Highly exposed persistently seronegative women have been reported to show mucosal anti-HIV antibody responses. ${ }^{7-10}$ There are very few reports of vaginal vaccination in women ${ }^{11-13}$ and, with the exception of one study ${ }^{11}$ that used inactivated poliovirus, they have involved cholera toxin subunit $\mathrm{B}$, a potent mucosal immunogen that does not require the use of an adjuvant.

We are investigating the potential of a recombinant HIV-1 clade C gp140 formulated in a poly-acrylic acid (Carbopol) gel to induce antibody responses in the lower female genital tract when applied at regular intervals equivalent to the time frame of the inter-menses interval of the human menstrual cycle.

\footnotetext{
${ }^{1}$ Centre for Infection, Division of Cellular and Molecular Medicine, St George's University of London, London, UK. ²Polymun Scientific Immunbiologische Forschung $\mathrm{GmbH}$, Vienna, Austria. ${ }^{3}$ Jefferiss Research Trust Laboratories, Wright Fleming Institute, Division of Medicine, Imperial College, London, UK. ${ }^{4} \mathrm{CAVD}$ Neutralizing Antibody Laboratory, Beth Israel Deaconess Medical Center, Harvard Medical School, Boston, Massachusetts, USA. Correspondence: MP Cranage (mcranage@sgul.ac.uk) 
Table 1 Assignment of animals to experimental groups

\begin{tabular}{|c|c|c|c|c|c|}
\hline Group & Treatment & $\begin{array}{c}\text { Concentration of } \\
\text { vaccine }\end{array}$ & $\begin{array}{l}\text { No. of immun. } \\
\text { cycles }\end{array}$ & \multicolumn{2}{|c|}{ Number of animals } \\
\hline A & gp140 & $100 \mu \mathrm{g}$ in $620 \mu \mathrm{l}$ & 1 & 6 & 4 \\
\hline C & Sham control & 0 & 1 & 6 & 4 \\
\hline $\mathrm{D}$ & gp140 & $65 \mu \mathrm{g}$ in $400 \mu \mathrm{l}$ & 1 & 6 & 0 \\
\hline $\mathrm{F}$ & gp140 & $306 \mu \mathrm{g}$ in $620 \mu \mathrm{l}$ & 3 & 6 & 4 \\
\hline$G$ & Gel control & 0 & 3 & 6 & 4 \\
\hline
\end{tabular}

gp, glycoprotein; immun., immunization

aMain group: necropsied 1 day after cessation of immunization.

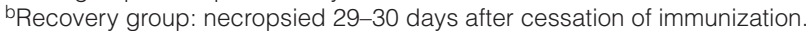

The choice of envelope protein was based on the high prevalence of HIV-1 clade C circulating in the world. Our approach builds on the concept of topically applied microbicides: self-administered anti-viral gels applied vaginally and designed for frequent use before each sexual encounter. Ultimately, it may be possible to combine vaginal immunization with the use of vaginal HIV microbicides. Here we show, in the rabbit, that repeated vaginal exposure to recombinant HIV-1 clade C envelope, formulated in a Carbopol gel, is well tolerated, and induces systemic and mucosal immunoglobulin $\mathrm{G}$ ( $\operatorname{IgG}$ ) antibody responses.

\section{RESULTS}

\section{A single cycle of intravaginal immunization induced serum} antibody

A total of 10 rabbits were inoculated intravaginally, each with $620 \mu \mathrm{l}$ Carbopol gel containing $100 \mu \mathrm{g}$ of CN54 gp140, on each of 9 occasions every 2 or 3 days over a 19-day period (Group A: Table 1). In addition, six rabbits were similarly inoculated with doses of $400 \mu \mathrm{l}$ of formulated antigen, equivalent to $65 \mu \mathrm{g}$ of antigen per dose (Group D). A further 10 animals received $620 \mu \mathrm{l}$ volumes of Carbopol gel alone (Group B) and 10 animals were sham inoculated (Group C).

All pre-treatment serum samples and those from control groups, at every time point, tested negative for CN54-specific IgG and IgA antibodies. Sera from animals in Group A tested 1 day after the final intravaginal inoculation (day 20) were all positive for IgG antibody, with titers ranging from 1,050 to 22,500 (Figure 1a). In animals no. 27, no. 28, no. 29, and no. 30, which were also tested 30 days after receiving the final dose of antigen (day 49), titers were either maintained or had increased (range $5,230-11,070)$; however, only animals no. 1 , no. 2 , no. 4 , and no. 5 of the 6 animals of Group D had sero-converted to IgG antibody by 1 day after cessation of intravaginal dosing, with titers ranging from 650 to 5,000. In animals no. 4 and no. 5, corresponding to those with the highest titers at day 20, specific IgG antibody was detectable, at low titer, by day 13 (Figure 1a). The median IgG antibody titer induced in Group A was significantly higher than that induced in Group D measured at day 20; 3,275 compared with 785, $P=0.02$; Mann-Whitney rank sum test. All serum samples, regardless of antigen dose, tested negative for specific IgA.

\section{A single cycle of intravaginal immunization induced serum HIV-1-neutralizing antibody}

Sera from Group A animals were analyzed for virus infectivity neutralizing activity (Figure 1b). No activity was detected preimmunization. After immunization, 6 of 10 vaccinates (animal no. 21 , no. 22 , no. 24 , no. 25 , no. 27 , and no. 30 ) showed anti-tier 1 clade C (MW965.26) neutralizing titers ranging from 20 to 148. Of the 4 recovery group animals, no. 27 and no. 30 had neutralizing activity at day 49 , but only in animal no. 30 was the neutralizing antibody detected at day 20. Interestingly, the rise in binding antibody titer over the same period (Figure 1a) was relatively modest. Only 3 of 10 animals (no. 22, no. 23, and no. 30) generated neutralizing antibody against tier 2 clade C 96ZM651.02 pseudovirus with very low titers ranging from 22 to 28 , and this was not directly associated with titers against tier 1 virus. An autologous CN54 Env pseudotype virus was not available.

\section{A single cycle of intravaginal immunization induced mucosally detectable antibody}

Eluates from vestibular and vaginal spears taken at necropsy from Group A animals and from control animals (Groups B and C) were first screened undiluted for gp140-specific IgG. All samples from Group A rabbits, including those from the four recovery group animals sampled 30 days after cessation of immunization, tested positive, with absorbance values ranging from 0.406 to 3.95 (Figure 2). Samples from the control groups were negative. There was no statistical difference between the median absorbance values obtained from paired samples taken from the vestibule and the vagina $(P=0.34$; Wilcoxon signedrank test).

Immunoglobulin A antibody was not detected on subsequent screening of vaginal and vestibule eluates; however, this analysis was done after an additional freeze-thaw cycle, which also resulted in an appreciable reduction in absorbance values for IgG antibody (data not shown). Nonetheless, in vaginal samples from seven rabbits, in which sufficient volume was available for testing, titers of gp 140-specific IgG ranged from 1 to 65 .

Mucosal eluates from Group D were subject to only a single freeze-thaw cycle. In animals no. 1, no. 2, no. 4, and no. 5, corresponding to those with serum IgG titers, specific $\operatorname{IgG}$ was 


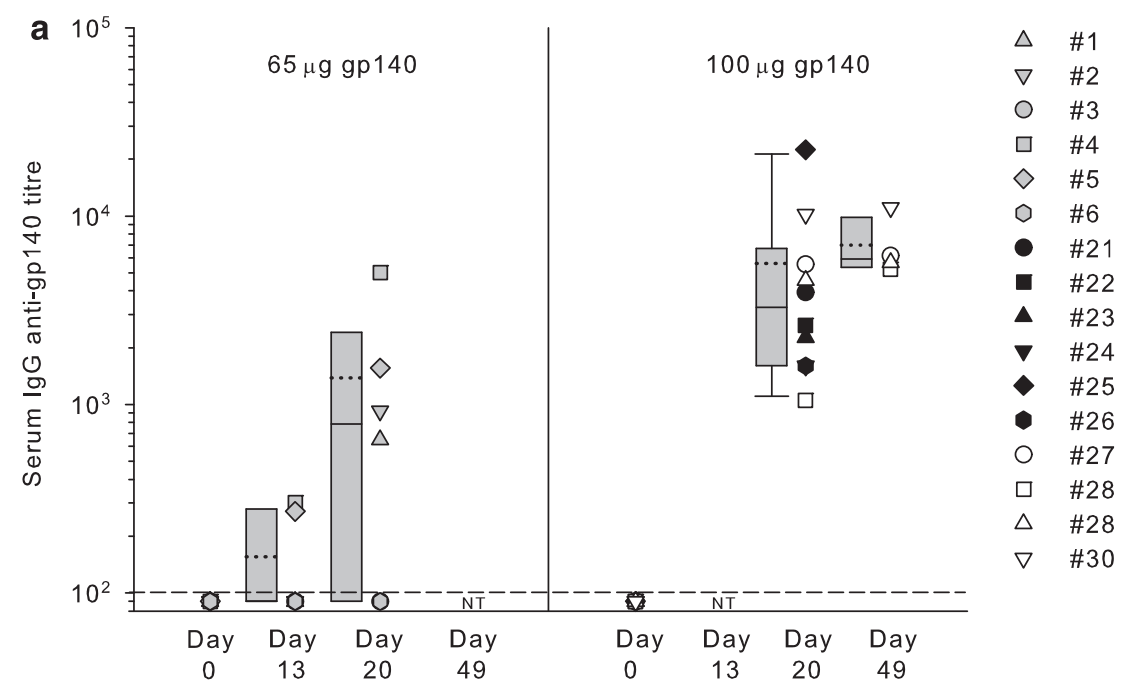

MW 965.26

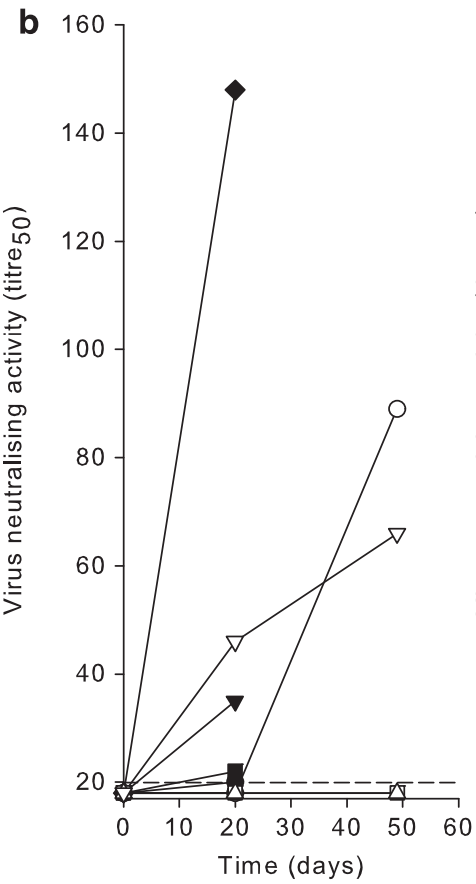

$96 Z M 651.02$

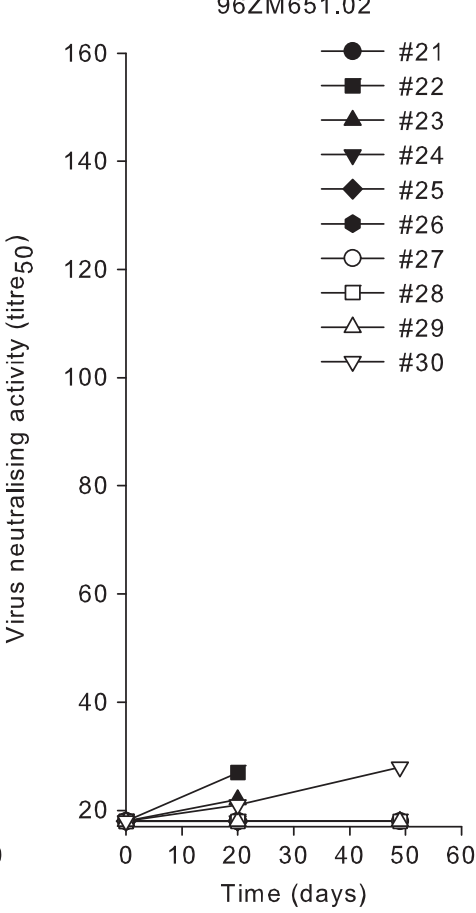

Figure 1 (a) Serum anti-gp140 (glycoprotein 140) immunoglobulin G ( $\mathrm{gGG}$ ) antibody titers after a single cycle of intravaginal administration of 9x65 $\mu \mathrm{g}$ doses of gp140 (Group D) or $9 \times 100 \mu \mathrm{g}$ doses of gp140 (Group A). The limit of detection is shown as a horizontal dashed line. Box plots show 25 th and 75th percentiles, error bars show 10th and 90th percentiles, (...) show mean and (-) show median titers of the groups. NT is not tested. (b) Serum virus infectivity neutralizing activity against human immunodeficiency virus (HIV)-1 clade C tier 1 (MW965.26) and tier 2 (96ZM651.02) pseudovirus detected in Group A animals.

detected in both vaginal and vestibule samples. Interestingly, despite the absence of serum IgA antibody, four animals (no. 2, no. 3 , no. 5 , and no. 6 ) had detectable IgA antibody in vaginal and/or vestibule samples, and in rabbits no. 3 and no. 6 , this was detected in the absence of mucosal or serum IgG antibody (Table 2). Volume limitation precluded further analysis.

\section{Two cycles of intravaginal immunization boosted serum antibody titers}

Next, the effects of multiple cycles of immunization and dose were investigated. All animals immunized with one cycle of
$9 \times 100 \mu$ g doses (Group E) made a serum IgG response as noted previously in Group A, and 9 of 10 animals receiving $306 \mu \mathrm{g}$ (Group F) also responded (Figure 3). There was no statistical difference between median titers in Groups $\mathrm{E}$ and $\mathrm{F}(P=0.47$; Mann-Whitney rank sum test). After a further cycle of immunization, individual antibody titers were boosted in all animals in each group, giving a significant increase in group mean titers ( $P=0.008$, Group E; $P<0.001$, Group F; paired $t$-test), but there was no difference between groups ( $P=0.91$; Mann-Whitney rank sum test). A further round of immunization did not significantly increase the mean titer in either group $(P=0.97$, Group E; 
$P=0.11$, Group F; paired $t$-test). Although the higher dose of antigen (Group F) did not significantly increase titer, there was a tighter clustering of "high responders" compared with Group E; furthermore, the third cycle of immunization in Group F boosted titer in animal no. 313 to reach the "high"-responder group. Anti-gp140 IgG titers were invariably lower after the 29-day "recovery phase" compared with those measured 1 day after cessation of the third cycle of immunization.

Nine animals in each group mounted low titer serum IgA antiCN54 gp140 responses (data not shown). In both groups, no IgA was detected after a single cycle of intravaginal immunization. However, after a second cycle of immunization, eight animals in each group had detectable IgA antibodies with titers ranging from 15 to 58 in Group E and from 13 to 56 in Group F at day 48. A further animal in each group seroconverted for IgA antibody after a third cycle of immunization. Animals with delayed sero-conversion corresponded to those with the lowest IgG titers after one cycle of immunization, whereas the two unrespon-

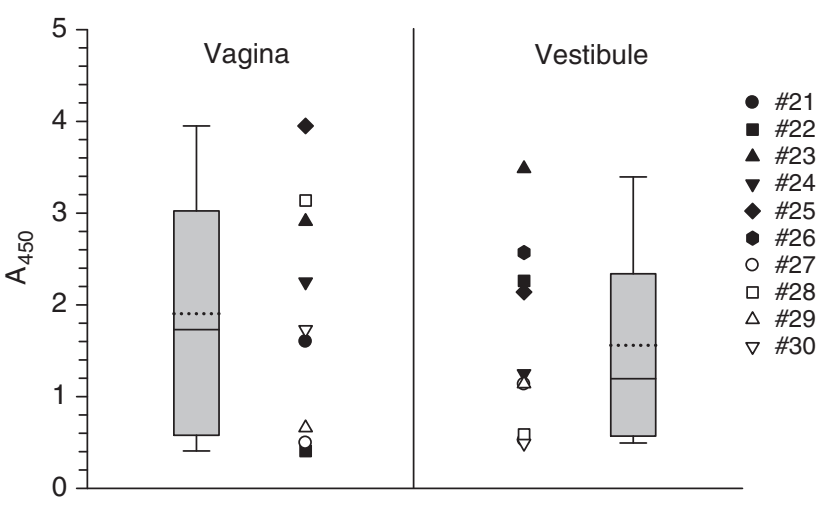

Figure 2 Detection of anti-gp140 (glycoprotein 140) immunoglobulin G (IgG) antibody in secretions from vagina and vestibule in rabbits after a single cycle of $9 \times 100 \mu \mathrm{g}$ doses of gp140 given intravaginally (Group A). Closed symbols show results for animals sampled 1 day after cessation of immunization (main group) and open symbols show results for animals sampled 29 days after cessation of immunization (recovery group). Mean (...) and median (-) absorbance values for each group are shown within box plots. Sample volume precluded determination of end-point titers and there was insufficient vaginal sample from animal no. 26 for testing. sive animals corresponded to the animals with the lowest $\operatorname{Ig} G$ titers after three cycles of immunization (animals no. 225 and no. 315). Two of four animals from Group E and all four animals from Group F had IgA antibody still detectable 29 days after cessation of immunization.

\section{Multiple cycles of intravaginal immunization augmented serum HIV-1-neutralizing antibody titers against tier 1 virus,} but did not induce activity against tier 1 envelope pseudotype Virus neutralizing titer was determined after two cycles of intravaginal immunization with either $100 \mu \mathrm{g}$ doses (Group E) or $306 \mu \mathrm{g}$ doses (Group F). Comparison of results for neutralization of tier 1 virus after a single cycle of immunization (Group A, Figure 1b) with that after the second cycle of immunization (Group E, Figure 4a) at $100 \mu \mathrm{g}$ doses revealed a highly significant difference $(P<0.001$; Mann-Whitney rank sum test). There was no significant difference, however, between virus neutralizing titer obtained after two cycles of immunization with $100 \mu \mathrm{g}$ or $306 \mu \mathrm{g}$ dosing $(P=0.97$; Mann-Whitney rank sum test) (Figures $4 \mathbf{a}$ and $\mathbf{b}$ ). There was insufficient sample volume for analysis after three cycles of immunization; however, titers determined in the "recovery" group animals indicated that neutralizing activity was present 29 days after cessation of the third cycle of immunization at levels that were not significantly different to those detected at the cessation of the second cycle of immunization ( $P=0.625$ for both groups; Wilcoxon signedrank test). Overall, anti-tier 1 virus neutralizing titers correlated with gp 140-binding antibody titers measured by ELISA (enzyme-linked immunosorbent assay) $(r=0.796 ; P<0.001$, Pearson product moment correlation); however, when analyzed by group, only Group E receiving $100 \mu \mathrm{g}$ doses of gp 140 showed significant correlation $(r=0.93 ; P<0.001)$, whereas data from Group F was poorly correlated $(r=0.5 ; P=0.06)$. Despite multiple immunizations, neutralization of tier 2 envelope pseudotype virus was not detected.

\section{Multiple cycles of intravaginal immunization augmented specific IgG titers detected in the genital tract, but did not induce an IgA-specific response}

Screening of undiluted mucosal eluates by ELISA revealed that all samples from Groups E and F, regardless of sampling site,

Table 2 Detection of anti-gp140 antibodies in genital tract secretions from rabbits immunized with a single cycle of nine intravaginal doses of $65 \mu \mathrm{g}$ gp140 (Group D): comparison with serum antibody titers.

\begin{tabular}{|c|c|c|c|c|c|c|}
\hline 4 & 700 & 145 & 5,000 & $<0.2$ & $<0.2$ & $<10$ \\
\hline 1 & 45 & 80 & 650 & $<0.2$ & $<0.2$ & $<10$ \\
\hline 5 & 27 & 70 & 1,560 & $<0.2$ & 0.37 & $<10$ \\
\hline
\end{tabular}

gp, glycoprotein; Ig, immunoglobulin.

End-point titers are shown except for IgA in secretions in which undiluted samples were analysed because of volume limitations 

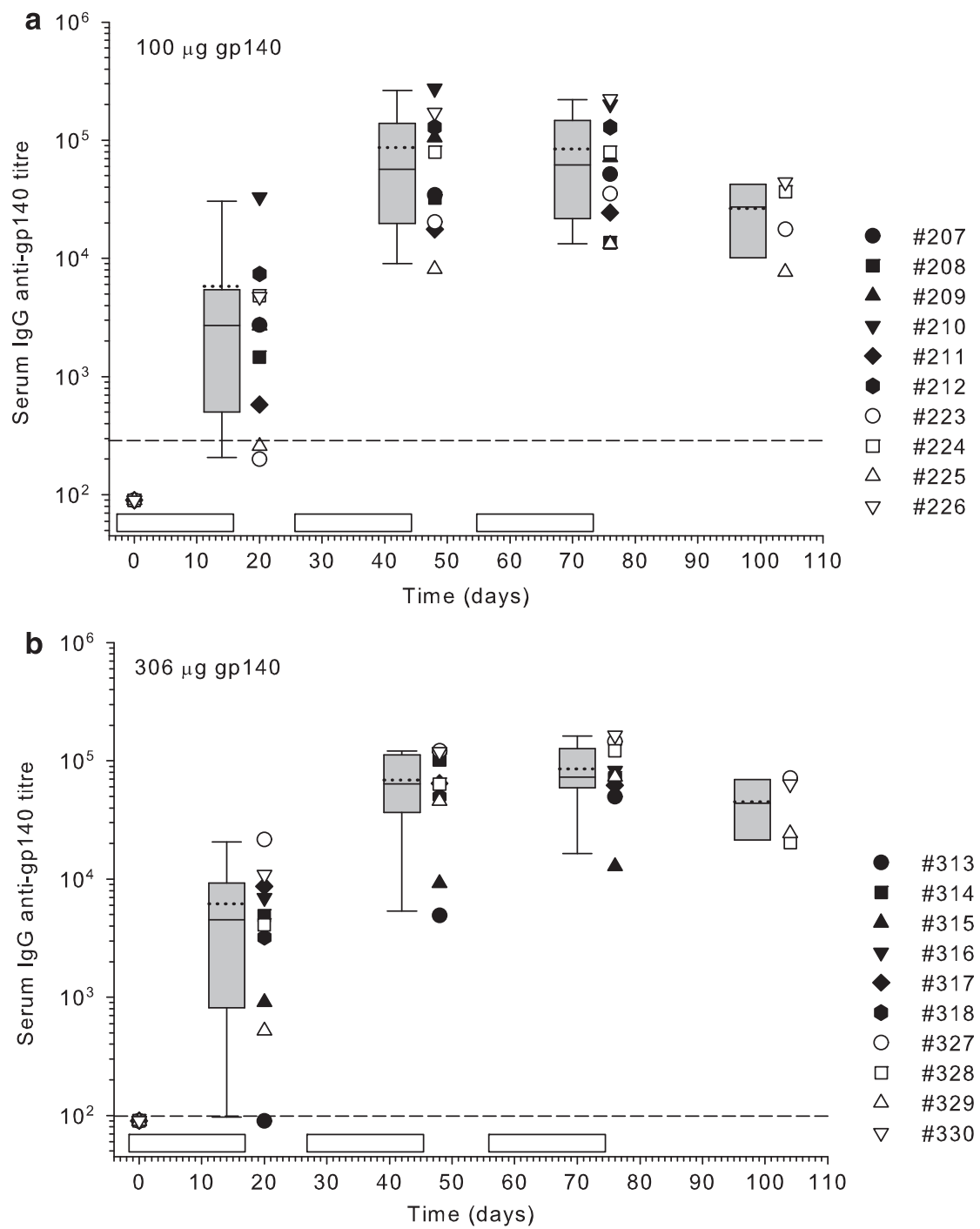

Figure 3 Serum anti-gp140 (glycoprotein 140) immunoglobulin G (IgG) antibody titers after multiple cycles of intravaginal immunization. Rabbits received $9 \times 100 \mu \mathrm{g}$ gp140 per cycle (Group E) (a) or 9×306 $\mu \mathrm{g}$ gp140 per cycle (Group F) (b). The limit of detection is shown as a horizontal dashed line. Immunization cycles are shown as open boxes. Group mean (...) and median (-) end point titers are shown within box plots.

gave saturating absorbance values for IgG antibody, whereas all samples from Group G (gel control) failed to bind. In contrast, IgA antibody was undetectable in any sample, despite the detection of total IgA. Titration of mucosal samples showed that IgG antibody titers normalized against the total IgG content of each sample (specific antibody activity) did not differ significantly between animals immunized with $100 \mu \mathrm{g}$ doses of gp140 (Group E) and those with $306 \mu \mathrm{g}$ doses (Group F) (Figure 5). Although normalized titers were somewhat lower in both vaginal and vestibular samples taken at day 104 compared with those taken immediately after cessation of immunization, this difference only reached statistical significance in vaginal samples from Group E ( $P=0.038$; Mann-Whitney rank sum test).

To determine whether the mucosally detected antibody was the result of serum exudation, the normalized anti-gp140 antibody titers for mucosal samples were compared pairwise with specific antibody activity in the corresponding serum sample. For both vaginal and vestibular samples, there was a highly statistically significant difference compared with serum $(P<0.001$; Wilcoxon signed-rank test). The proportion of specific antibody detected in mucosal samples was always higher than that detected in serum, being on average 8.5-fold higher in vaginal samples (range 1.4 to 25 ) and 9.0-fold higher in vestibular samples (range 2-22.8).

\section{The serum antibody response induced by multiple cycles of intravaginal vaccination was predominantly against the gp120 region of the gp140 antigen}

To determine the breadth of antigen recognition after intravaginal immunization, sera taken after three cycles of immunization or, where there was insufficient volume, after two cycles of immunization, were first screened on pools of peptides, available to the project, covering the predicted amino acid sequence of the entire gp140 of a prototypic clade C virus, 96ZM651. Frequency of recognition varied markedly and only 5 of 20 sera recognized peptides in the gp41-ED (external domain) of gp140 

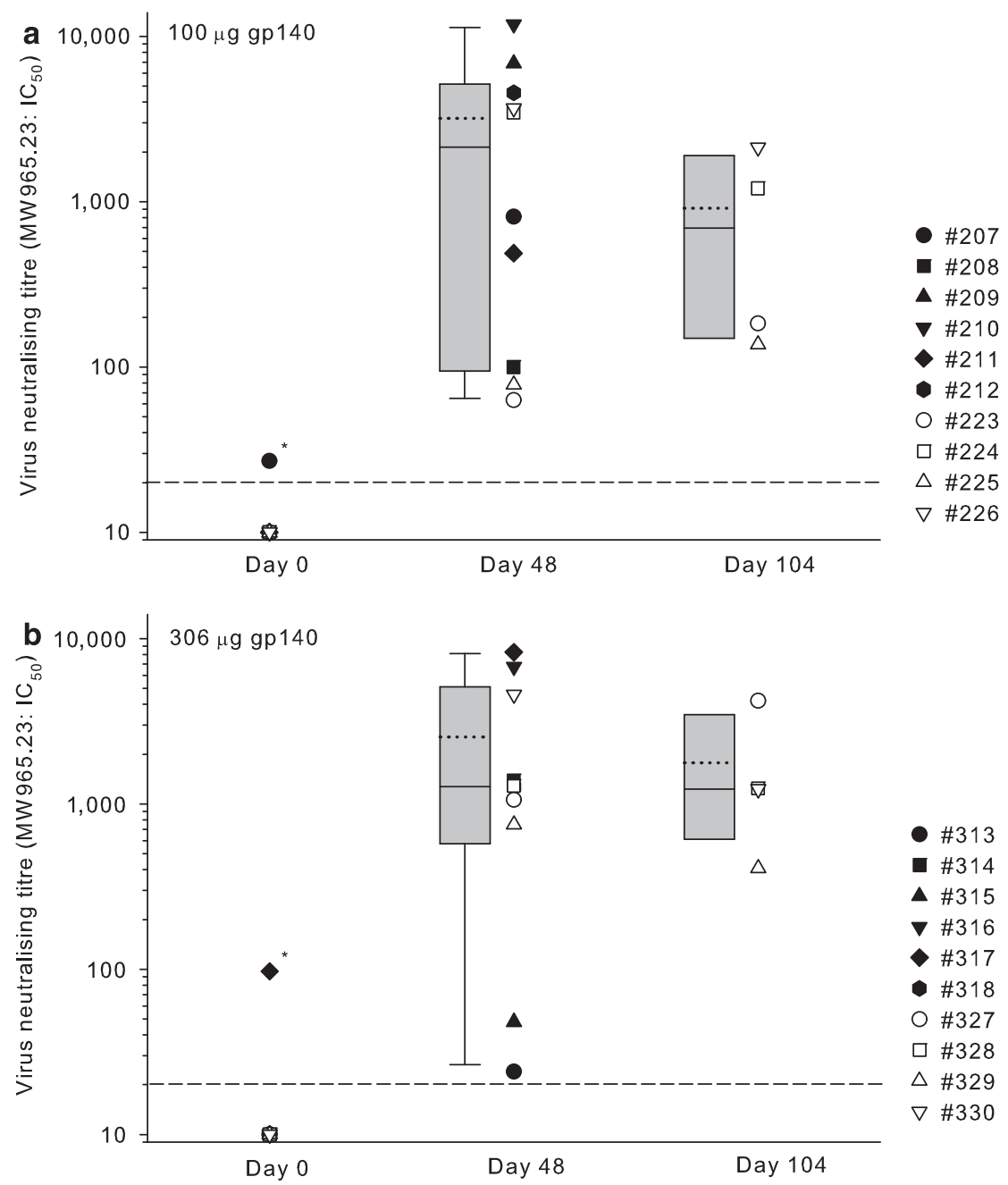

Figure 4 Virus infectivity neutralizing activity before immunization, 1 day after cessation of two cycles of intravaginal immunization, and 29 days after a third cycle of intravaginal immunization using $9 \times 100 \mu \mathrm{g}$ doses per cycle (Group E) (a) or $9 \times 306 \mu \mathrm{g}$ doses per cycle (Group F) (b). Individual 50\% inhibitory concentration $\left(\mathrm{IC}_{50}\right)$ titers are shown for neutralization of human immunodeficiency virus (HIV)-1 clade $\mathrm{C}$ tier 1 virus. Group mean (......) and median (-) titers are shown within box plots. ${ }^{*}$ Indicates nonspecific neutralization determined by reactivity with MuLV (murine leukemia virus)envelope pseudotyped virus.

(Figure 6a). In general, the number of pools recognized was positively associated with the anti-gp140-binding titer, but there was no absolute correspondence; neither was there a direct association between breadth of recognition and the number of cycles or immunizing dose. The number of pools recognized after two cycles of immunization was, in general, positively associated with the anti-tier 1 virus neutralization titer.

Next, sera that screened positive against peptide pools were tested against each individual peptide component of the pool. The frequency of recognition was compared with results obtained with sera from non-clade-typed HIV-positive individuals (Figure 6b). Strikingly, all immunized rabbits recognized the $\mathrm{V} 3$ region, whereas this was recognized in only 20 percent of the human $\mathrm{HIV}^{+}$sera. In general, regions within $\mathrm{C} 1$ were recognized after immunization, and the distribution of binding suggested the presence of multiple epitopes. The V1 and V2 regions were infrequently recognized by the rabbit sera and, surprisingly, were neither recognized by human sera, possibly because of clade

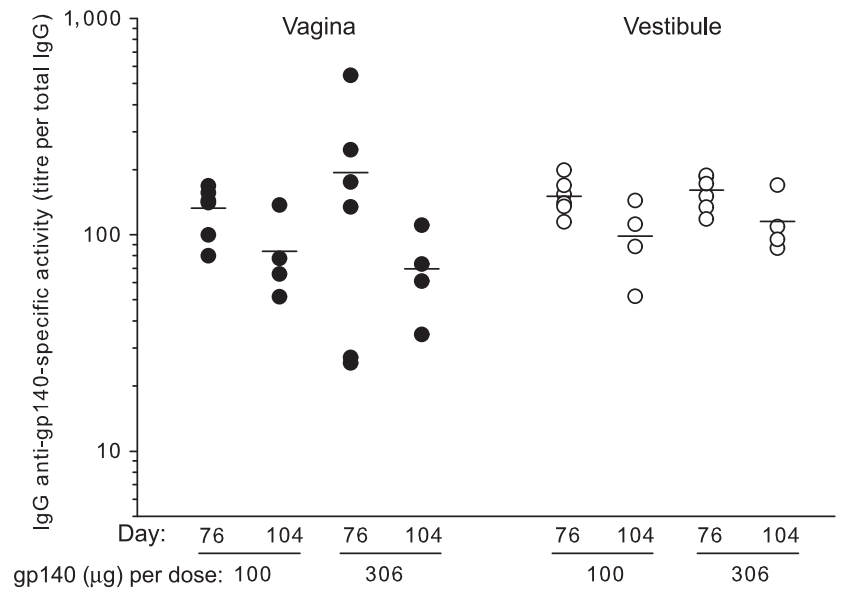

Figure 5 Detection of anti-gp140 (glycoprotein 140) immunoglobulin G (IgG) antibody in mucosal secretions after three cycles of intravaginal immunization. Day 76 represents 1 day after the third cycle of immunization and day 104 represents 29 days after cessation of immunization (recovery group). Bars indicate mean titer. 
a

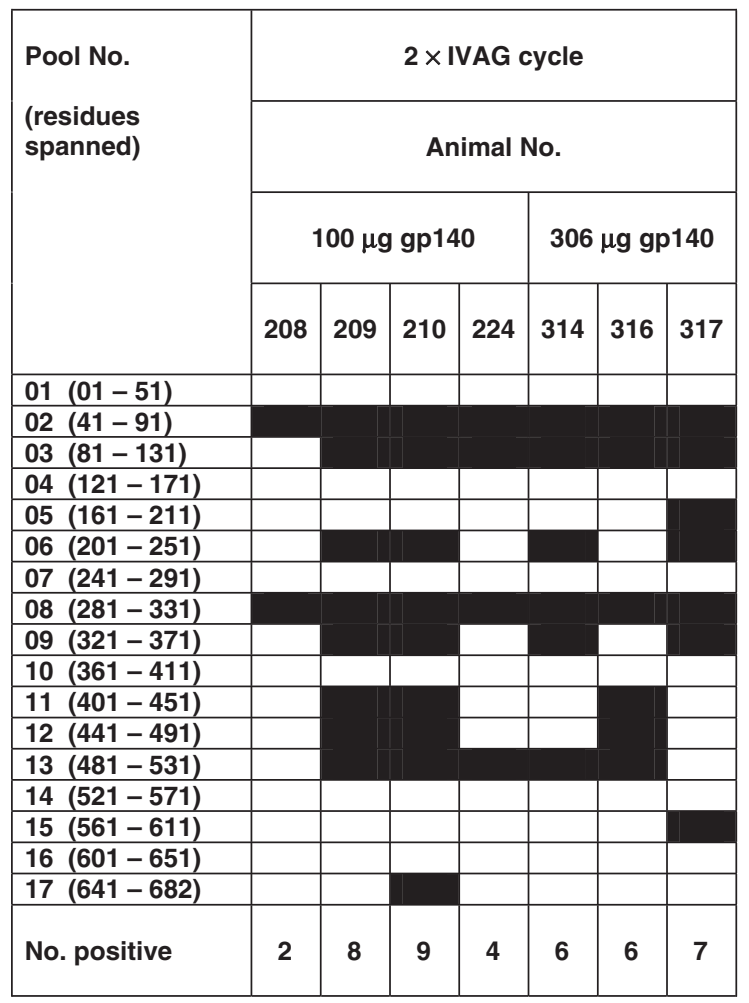

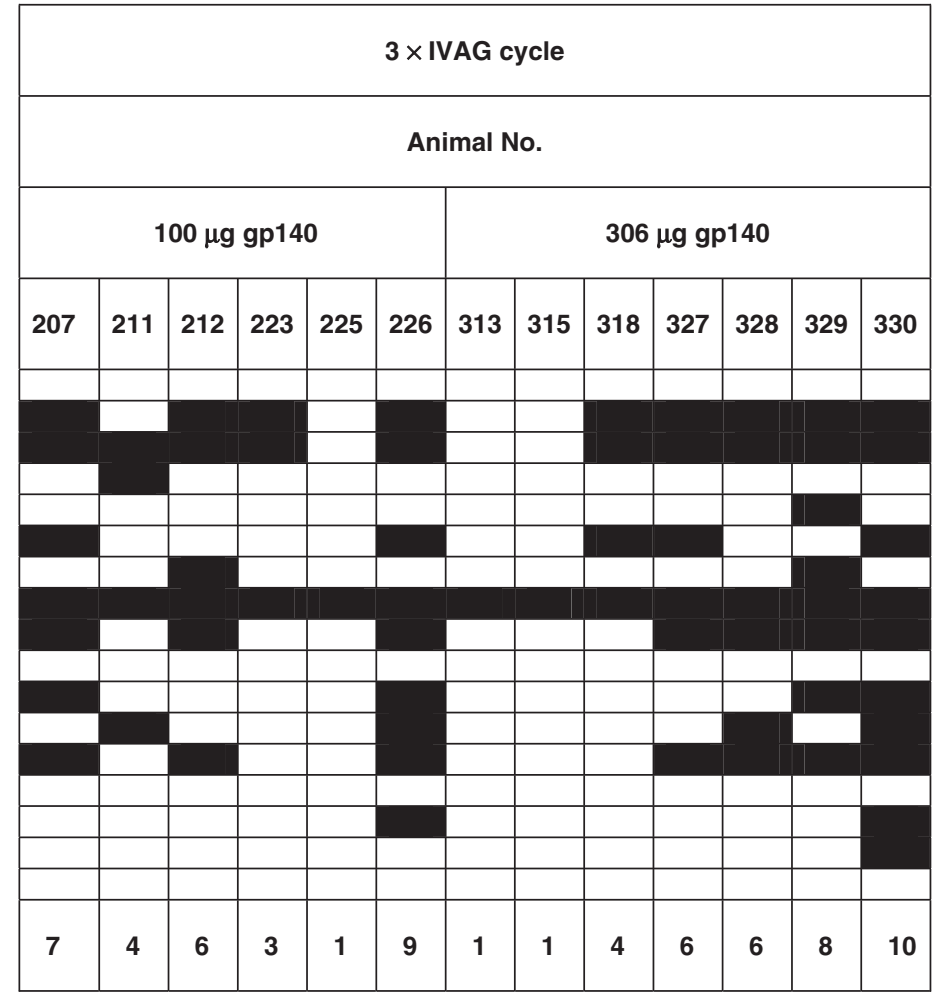

b

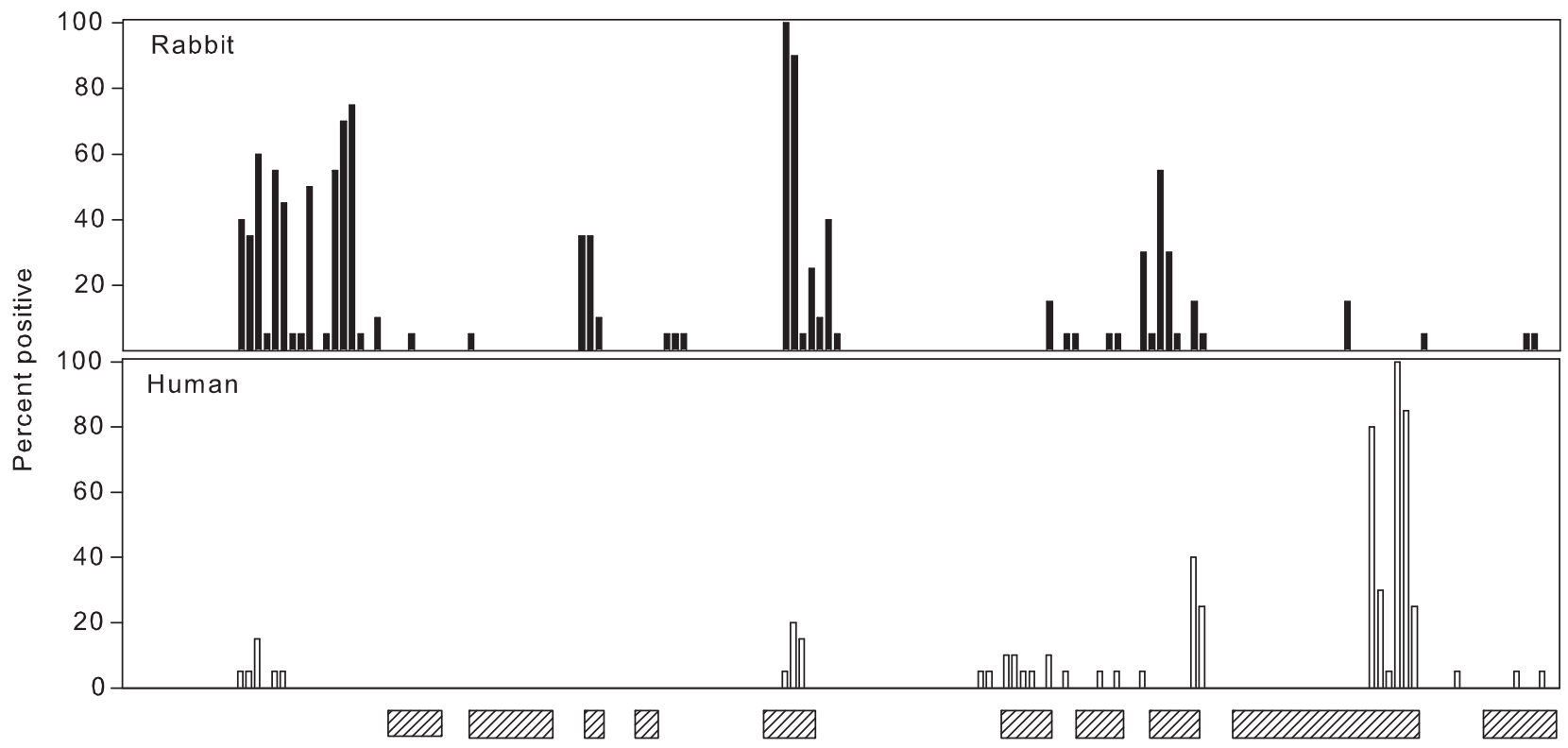

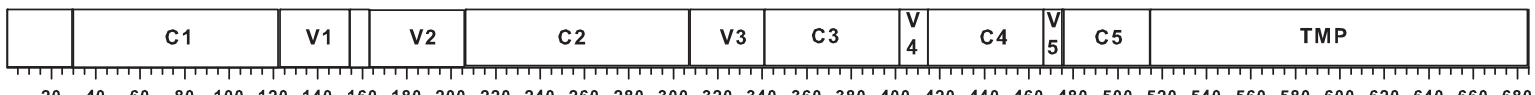
Residue No.

Figure 6 Frequency of recognition of overlapping 15mer 96ZM651 Env peptides by sera from intravaginally immunized rabbits. (a) Shows the frequency of reactivity with pools of peptides consisting of 1015 mers, each overlapping by 11 amino acids. Black boxes indicate binding at $\geqslant 0.2$ absorbance units.

(b) Frequency of reactivity of pool-positive sera against individual peptides. The frequency distribution for immunized rabbits is compared with that obtained with 20 sera from non-clade-typed $\mathrm{HIV}^{+}$individuals. Shaded boxes denote regions of known antibody recognition ${ }^{35}$ aligned against structural regions of the HIV-1 HXB2 clade B-predicted amino acid sequence. Residue numbers refer to the prototypic clade C 96ZM651 envelope-predicted amino acid sequence.

specificity. Only a minority of sera bound to peptides in the $\mathrm{V} 4, \mathrm{C} 4$, and V5 regions, although a somewhat higher frequency of recognition was seen with rabbit sera recognizing the $\mathrm{C} 5$ region. All human sera recognized the highly immunodominant domain in the gp41-ED (residues 598-609) and the majority recognized the region 586-597 upstream of 


\begin{tabular}{|c|c|c|c|c|c|c|c|c|c|}
\hline \multirow[b]{2}{*}{ Animal no. } & \multirow[b]{2}{*}{ Group } & \multicolumn{4}{|c|}{ Serum 1/5 } & \multicolumn{4}{|c|}{ Serum 1/20 } \\
\hline & & Day 20 & Day 48 & Day 76 & Day 104 & Day 20 & Day 48 & Day 76 & Day 104 \\
\hline 119 & $G$ & ++ & ++ & ++ & ++ & ++ & ++ & ++ & ++ \\
\hline 207 & $\mathrm{E}$ & ++ & ++ & $\#$ & NA & ++ & ++ & + & NA \\
\hline 208 & & ++ & + & $+/-$ & NA & ++ & ++ & ++ & NA \\
\hline 209 & & ++ & - & - & NA & ++ & $+1-$ & $+/-$ & NA \\
\hline 210 & & ++ & - & - & NA & ++ & $+1-$ & $+1-$ & NA \\
\hline 211 & & ++ & ++ & + & NA & ++ & ++ & ++ & NA \\
\hline 212 & & ++ & - & - & NA & ++ & + & + & NA \\
\hline 223 & & ++ & ++ & - & + & ++ & ++ & ++ & ++ \\
\hline 224 & & ++ & $+1-$ & - & $+1-$ & ++ & ++ & ++ & ++ \\
\hline 225 & & ++ & ++ & ++ & ++ & ++ & ++ & ++ & ++ \\
\hline 226 & & ++ & - & - & - & ++ & + & - & + \\
\hline 313 & $\mathrm{~F}$ & ++ & ++ & + & NA & ++ & ++ & ++ & NA \\
\hline 314 & & ++ & - & - & NA & ++ & + & + & NA \\
\hline 315 & & ++ & ++ & ++ & NA & ++ & ++ & ++ & NA \\
\hline 316 & & ++ & - & - & NA & ++ & $+1-$ & - & NA \\
\hline 317 & & ++ & - & - & NA & ++ & ++ & ++ & NA \\
\hline 318 & & ++ & + & - & NA & ++ & ++ & ++ & NA \\
\hline 327 & & ++ & - & ND & + & ++ & + & ND & ++ \\
\hline 328 & & ++ & + & ND & $+1-$ & ++ & ++ & ND & ++ \\
\hline 330 & & ++ & - & ND & - & ++ & ++ & ND & ++ \\
\hline
\end{tabular}

,++ gp140 trimer band at intensity equal to that after reaction with pre-immunization serum; +, +/ , , , relative intensity of gp140 trimer band; \# result undetermined because of gel artefact; gp, glycoprotein; NA, not applicable; ND, not done.

Sera were incubated with unlabelled gp140 vaccine preparation and residual unreacted gp 140 oligomers separated by native gel electrophoresis. After electroblotting, separated proteins were detected with labeled human monoclonal antibodies. Sera with high reactivity against nondenatured trimeric gp140 formed immune complexes that were unable to enter the native gel and therefore scored negative.

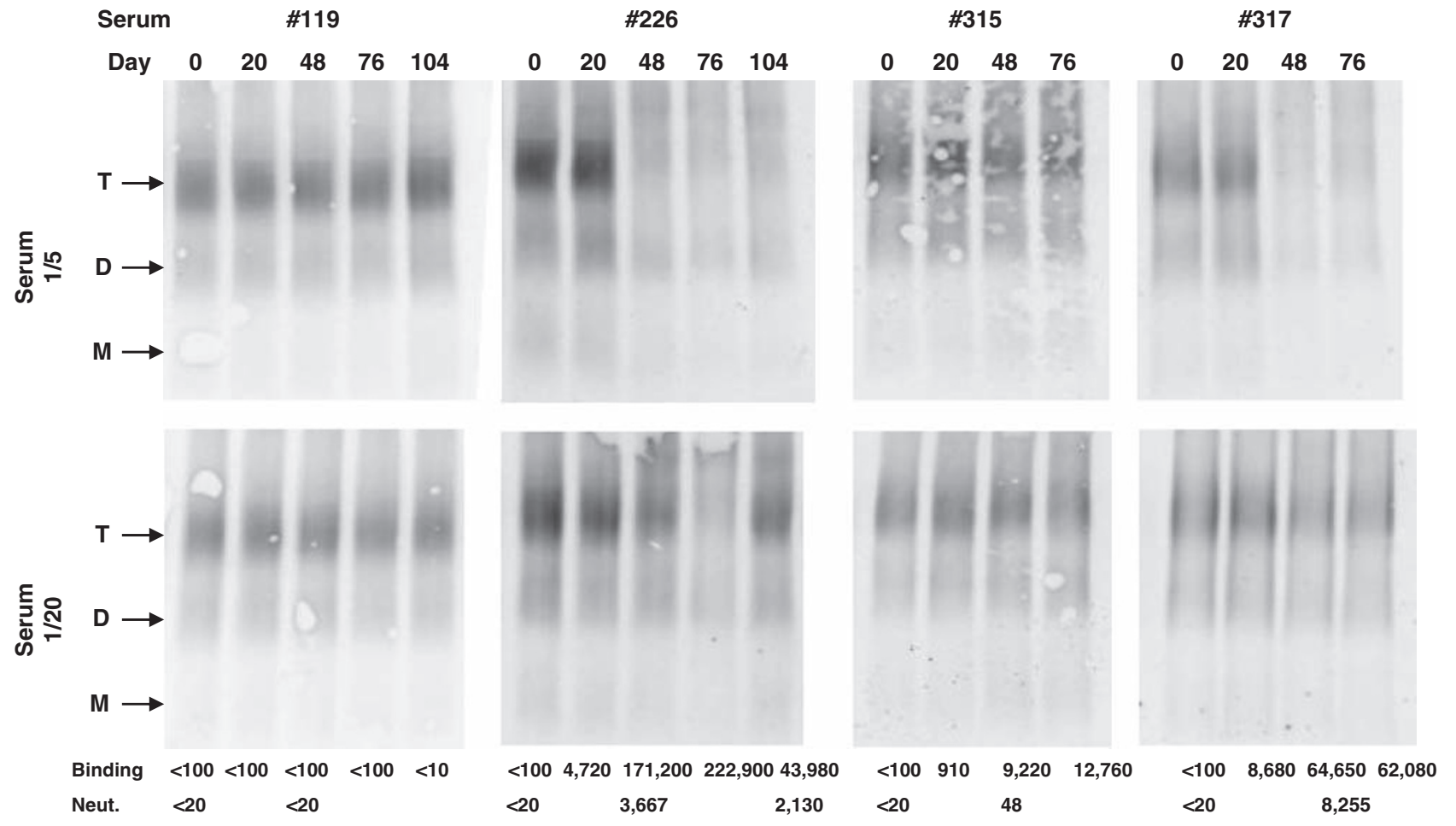

Figure 7 Representative results from analysis of antibody reactivity with oligomeric gp140 under nondenaturing conditions. T indicates trimer, $\mathrm{D}$ indicates dimer, and M indicates monomer. Binding indicates end-point IgG anti-CN54 gp140 titer determined by enzyme-linked immunosorbent assay (ELISA). Neut. indicates infectivity neutralizing activity (50\% inhibitory concentration, $\left.I C_{50}\right)$ against tier 1 pseudovirus. 
the immunodominant epitope. Notably, this region was not recognized by sera from immunized rabbits.

To determine whether antibodies specifically able to bind to higher order gp140 structures were elicited, sera from immunized rabbits were analyzed for their oligomer binding under nondenaturing conditions. Reactivity with CN54 gp140 trimer was detected only after multiple immunization cycles (Table 3, Figure 7). In general, the readout from the oligomer assay reflected the titer of IgG antibody determined by ELISA (Figure 3). When tested at $1 / 5$, all sera with anti-tier 1 neutralization titers above 1,000 prevented or reduced detection of trimeric gp140. At 1/20, however, the threshold of reactivity was less defined. A total of 7 of 11 sera with anti-tier 1 neutralization titers $>1,300$ prevented or reduced detection of trimeric gp 140; however, sera from rabbits no. 317, no. 330, no. 327 (day 104), and no. 224 failed to react despite neutralizing titers of 8,255 , $4,576,4,212$, and 3,460 , respectively. Three compared with two cycles of immunization and $100 \mu \mathrm{g}$ compared with $306 \mu \mathrm{g}$ doses were not found to differentially confer advantage in terms of antibody recognition of trimeric gp140. The results also show that the majority of the antigen, at least in native gel buffer, was in the trimeric form, with some dimer and only trace amounts of monomer detectable.

\section{Carbopol and Carbopol-gp140 formulation were well tolerated} Treatment of rabbits with the CN54 gp140-Carbopol gel formulation or Carbopol gel alone, regardless of dosing schedule, resulted in no signs of systemic toxicity. Clinical condition, body weight, and food consumption all remained unaffected. No treatment-related ophthalmic lesions were seen, and hematological and blood chemistry analyses revealed no treatmentrelated effects. Likewise, no macroscopic findings or differences in organ weight, attributable to treatment, were found at necropsy.

Analysis of histological changes in the vagina indicated that cumulative scores for all animals, regardless of the concentration or frequency of administration of the product, all fell within the acceptable range for human vaginal irritation potential.

\section{DISCUSSION}

This is the first demonstration that soluble recombinant HIV-1 gp140 is immunogenic on repeated intravaginal immunization in the absence of a mucosal adjuvant such as those derived from bacterial enterotoxins. Moreover, the formulation was well tolerated in the rabbit vaginal irritancy model, even after multiple cycles of high-dose administration. The data indicate that the safety profile is acceptable for human clinical trial, in which the same antigen formulation will be administered nine times through the inter-menses interval to cover the optimum time for immune induction and response. Although the study design in rabbits was not optimized for the analysis of immune response dynamics, it was encouraging that a single cycle of intravaginal administration of the formulated antigen elicited serum antibodies and IgG antibodies detected in mucosal secretions. Carbopol gel was used as a carrier, as it is approved for vaginal use in women, being a component of licensed vaginal products and experimental HIV microbicide formulations. Although we cannot rule out that the Carbopol contributed to the generation of the responses reported here (although Carbopol showed no adjuvanticity when used vaginally in combination with gp 140 in mice; Q Sattentau, personal communication), it probably acted as an inert carrier aiding retention of the gp140 antigen in the vaginal vault. Other formulations containing multiple polymer components, including a mucoadhesive and hydrophilic matrix-forming polymer together with a polymeric component capable of absorbing vaginal fluid designed to enhance retention, are also giving promising results. ${ }^{14}$

A single immunization cycle with $100 \mu \mathrm{g}$ doses of gp 140 was clearly superior to $65 \mu \mathrm{g}$ doses, administered at the same concentration, in terms of eliciting serum IgG antibody, and a significant booster effect was seen after a further cycle of immunization; however, increasing the dose of antigen to $306 \mu \mathrm{g}$ per administration failed to increase the overall titer as did administration of a further cycle of immunization at either dose, suggesting that the saturating dose of antigen had been reached for these conditions. Only after multiple cycles of intravaginal immunization was serum IgA antibody detected, but this was of low titer and not present in all animals. Surprisingly, perhaps, IgA antibody was detected in vaginal and vestibular secretions only in the animals given a single cycle of $65 \mu \mathrm{g}$ doses and only in some animals at a low level. Although technical limitations precluded analysis of Group A, there was no evidence of local IgA antibody production in animals receiving multiple cycles of immunization at the higher doses of antigen. In contrast, IgG antibodies were readily detected in mucosal secretions and reached highest titers after multiple cycles of immunization. In another study in progress using a similar formulation, vaginal IgA was detected in mice but not in rabbits ( $Q$ Sattentau, personal communication). These results contrast those described after intravaginal immunization of rabbits with HPV 6bL1 DNA, in which IgA antibodies were elicited in the absence of IgG antibody. ${ }^{15}$ However, this latter study differed from that described here in using plasmid DNA, the co-administration of cholera toxin, and the use of mucosal injection within the vagina. The lack of mucosal IgA in the present study may be associated with the soluble nature of the antigen used. ${ }^{16,17}$ Furthermore, at least in humans, cervico-vaginal fluid contains more IgG than $\operatorname{IgA},{ }^{18}$ and the endocervix contains a high proportion of IgG antibody-secreting cells. ${ }^{19}$ In the present study, the lack of a rabbit IgA standard precluded direct analysis of the relative concentrations of total IgG and IgA. However, the higher specific activity of $\operatorname{IgG}$ antibody detected in the mucosal samples compared with that detected in serum suggests that, at least in part, the antibody may have been synthesized locally by B cell antibody-secreting cells in the mucosa. Moreover, antibody detected in the lower reproductive tract may have been produced at multiple sites, including those in the upper tract, because of the propensity of material to be transported upstream after intravaginal administration. It is also likely that, as has been reported in HIV infection, ${ }^{20}$ serum transudation also contributed to the antibody detected locally. 
The suitability of the rabbit for the assessment of intravaginal immunogenicity, as opposed to irritancy determination, is unknown. The anatomy of the lower female genital tract of the rabbit is a complicating factor as the upper two-thirds of the vagina has a nonstratified epithelium that may be more susceptible to antigen uptake. Moreover, the physiology of the rabbit reproductive cycle is markedly different from humans. Rabbits are induced ovulators and, therefore, are not suitable for modelling directly the effects of the human reproductive cycle on immune response generation. Indeed species differences may, at least in part, account for the reported contradictory observations regarding the efficacy of the female genital tract as a site for induction of antibody responses. ${ }^{13}$ Nonetheless, the detection of antibody in both the circulation and in the genital tract shows that the concept of repeated or sustained mucosal exposure to a putative vaccine antigen is promising.

Not only is there a requirement for sustained production of antibody into the female genital tract but this antibody must also have potent virus infectivity neutralizing activity if sterilizing immunity is to be achieved. The envelope antigen used in the present study has shown remarkable trimeric stability in vitro (D Katinger, personal communication), and this is considered highly desirable to focus the antibody response onto structures that may more accurately mimic those seen within the virus envelope spike. ${ }^{21}$ Intravaginal immunization clearly induced virus neutralizing activity that was boosted significantly by two cycles of immunization. Notably, however, activity was only seen against the more easily neutralized envelope. The almost complete lack of activity against tier 2 envelope pseudotype, even after three rounds of immunization, was disappointing but it is well recognized that the envelope spikes of HIV primary isolates are hidden from antibody recognition by a number of mechanisms including glycan shielding, ${ }^{22,23}$ the presence of sequence variable loops ${ }^{24}$ and conformational masking, ${ }^{25}$ and even an intrinsic resistance of surface external regions of gp120 to allow access of antibody to artificially introduced tags. ${ }^{26}$ Indeed it was interesting that rabbits produced antibodies that recognized the linear epitopes within the V3 region. This region is considered occluded on many primary isolates and this may, at least in part, explain the lack of neutralizing activity in these sera against the tier 2 envelope pseudotype virus. If so, then the recognition of this region after immunization may indicate that the trimeric structure of the antigen used here was markedly different from the virus spike. Furthermore, major differences were noted when reactivity of sera from immunized rabbits was compared with sera from HIV-infected individuals, most notably in the failure of rabbits to recognize the highly immunodominant region of gp $41 .{ }^{27}$ Although in this study a highly stable trimeric gp 140 was used, the immunogenic profile of this envelope could be influenced by several factors including formulation within the Carbopol gel, enzymatic degradation in vivo, and the use of a recombinant molecule in which a soluble molecule was created by termination before the membrane anchor. Nonetheless, the native binding assay confirmed directly that antibodies from immunized rabbits were able to bind to the trimeric gp140 used as the immunogen. This type of assay has been used as a surrogate for monoclonal antibody and polyclonal seramediated virus neutralization. ${ }^{28,29}$

The lower female genital tract has been described as a poor inductive site for adaptive immunity in the absence of strong adjuvants. ${ }^{18}$ In this context, our observation of systemic and mucosal immune responses after repeated vaginal exposure to recombinant HIV-1 clade C envelope, in a gel formulation without adjuvant, is encouraging. Ongoing studies in small animals, humans, and macaques will determine whether such responses to gel formulations can be improved after priming through different routes (intramuscular or intranasal, for example). The lack of detectable inflammation suggests that such gel-based formulations would be unlikely to enhance HIV transmission and that they would be compatible for co-delivery with candidate microbicides, designed for frequent use before each sexual encounter. The co-formulation with a topical microbicide might facilitate serial boosting of specific immune responses, supplementing the antiviral effect of the microbicide and reducing the chance of viral breakthrough against either intervention strategy.

\section{METHODS}

HIV gp140. A clade C envelope clone p97CN54 was obtained originally from HIV isolated from a Chinese patient ${ }^{30,31}$ and was made available by H Wolf and R Wagner, University of Regensburg, Germany. Trimeric gp140 (gp120 plus the ED of gp41), designated as CN54 gp140, was produced as a recombinant product in $\mathrm{CHO}$ cells and manufactured to good manufacturing practice specification by Polymun Scientific, Vienna, Austria.

Animals. New Zealand white rabbits (10-14-week-old) were supplied from an accredited closed colony, and the animals were housed at, and all in vivo procedures carried out by, a contract research organization in compliance with the United Kingdom Animals (Scientific Procedures) Act 1986 and associated Codes of Practice for the Housing and Care of Animals.

Intravaginal inoculation. Animals were assigned to experimental groups (Table 1). Immediately before inoculation, gp140 was formulated at the required concentration in Carbopol gel consisting of (w/w) water, $97.81 \%$; benzyl alcohol, 1.09\%; Carbopol 974P, 0.92\%; sodium hydroxide, $0.18 \%$. A total of $620 \mu \mathrm{l}$ or $400 \mu \mathrm{l}$ of the mixture was administered through a soft rubber catheter inserted $\sim 6 \mathrm{~cm}$ into the vagina. For any one cycle of intravaginal inoculation, animals received formulated product on nine occasions at days $1,3,5,8,10,12,15,17$, and 19 to mimic immunization through a human menstrual cycle. Necropsies were performed either 1 day after the final inoculation (main group) or 4 weeks later (recovery group). This timing interval was determined by the requirement for assessment of vaginal irritancy.

Assessment of systemic toxic potential and vaginal irritancy. Animals were inspected regularly for evidence of reaction to treatment or ill health. Before each intravaginal administration, animals were observed for signs of vulval irritation, discharge, or bleeding from the vagina. Body weights were recorded regularly. Samples were taken regularly for hematology and blood chemistry. At necropsy, special attention was given to the examination of the vagina and cervix, and detailed histopathology was carried out on transverse samples $(\sim 10 \mathrm{~mm})$ collected from the cervical and urethral ends of the vagina. Histological changes in the vagina were scored using methods modified from those of Eckstein et al. ${ }^{32}$ 
Blood and genital tract secretions. Peripheral blood was collected for serum before study commencement and after intravaginal vaccinations. Secretions from the vagina and the vestibule were sampled using WeckCel surgical spears (Medtronic Ophthalmics, Jacksonville, FL) postmortem from tissues that had been removed for this specific purpose, so as not to interfere with histological analysis. A sterile spear was placed on the mucosal surface of the vagina for $2 \mathrm{~min}$ to absorb any secretion. A second spear was placed on the mucosal surface of the vestibule. Secretions were eluted from the sponges by placing each spear into a Spin-X tube (Corning, Schiphol-Rijk, The Netherlands) containing $300 \mu$ l extraction buffer (protease inhibitor cocktail set 1, Calbiochem Merck, Beeston, UK). Samples were frozen at $-80^{\circ} \mathrm{C}$ and centrifuged before testing.

Enzyme-linked immunosobent assay. Binding antibodies against recombinant CN54 gp140 were measured using a standardized ELISA. CN54 gp140 was coated onto 96-well plates (medium binding, Greiner Bio-One, Stonehouse, UK) at $5 \mu \mathrm{g} \mathrm{ml}^{-1}$ in phosphate-buffered saline (PBS) for $1 \mathrm{~h}$ at $37^{\circ} \mathrm{C}$. Plates were washed four times in PBS containing $0.05 \%$ Tween 20 (PBS-T) before blocking with PBS-T containing $10 \%$ fetal bovine serum for $1 \mathrm{~h}$ at $37^{\circ} \mathrm{C}$. After further washing, sera diluted in PBS-T or mucosal eluates were added and incubated for $1 \mathrm{~h}$ at $37^{\circ} \mathrm{C}$. For IgG, bound antibody was detected with mouse monoclonal anti-rabbit IgG ( $\gamma$ chain-specific) peroxidase conjugate (A1949, Sigma Aldrich, Gillingham, UK). Normal rabbit serum was used as a negative control. Standard curves for IgG detection were derived using rabbit antiserum to HIV-1 GB8 gp120 (Programme EVA, Centre for AIDS Reagents, National Institute for Biological Standards and Control, ARP440.1/R546). IgA antibody was detected with biotin-conjugated goat IgG fraction of goat antiserum to rabbit IgA ( $\alpha$ chain-specific ABN116B, Autogen Bioclear, Calne, UK) followed by avidin conjugated to horseradish peroxidase (A7419, Sigma Aldrich). The goat antiserum was generated using purified polyclonal IgA from pooled rabbit serum and, therefore, would be expected to react against all rabbit IgA isotypes.

For linear epitope analysis, plates were coated with 15 mer peptides overlapping 11 residues, spanning a prototypic clade C gp140 sequence $(96 \mathrm{ZM} 651),{ }^{31}$ either in pools of 10 or singly.

Virus infectivity neutralization assay. Neutralizing antibody responses were measured using a luciferase-based assay in TZM.bl cells as previously described. ${ }^{33}$ This assay measures the reduction in luciferase reporter gene expression in TZM.bl cells after a single round of virus infection. The $50 \%$ inhibitory dose $\left(\mathrm{ID}_{50}\right)$ titer was calculated as the serum dilution that caused a 50\% reduction in relative luminescence units compared with the virus control wells after subtraction of cell control relative luminescence units. Stocks of Env-pseudotyped viruses were prepared by transfection of $293 \mathrm{~T} / 17$ cells as previously described. ${ }^{33}$

gp140 oligomer-binding assay. CN54 gp140 at a concentration of $530 \mu \mathrm{g} \mathrm{ml}^{-1}$ was mixed with $1 / 5$ or $1 / 20$ serum at a ratio of $1: 1$. After incubation overnight at $4{ }^{\circ} \mathrm{C}$, samples were applied to native tris-acetate polyacrylamide gels (Invitrogen, Paisley, UK). Separated proteins were electroblotted onto nitrocellulose membranes, and after blocking with 3\% $(\mathrm{w} / \mathrm{v})$ skimmed milk powder in PBS-T, transferred proteins were detected using a combination of human monoclonal antibodies: ${ }^{34} 1 \mathrm{~F} 7,3 \mathrm{~B} 7$, Hansi, $3 \mathrm{D} 6,5 \mathrm{~F} 3,4 \mathrm{~B} 3$, and $4 \mathrm{D} 4$ each at a concentration of $\sim 1 \mu \mathrm{g} \mathrm{ml}^{-1}$ followed by goat anti-human IgG-fluorescein isothiocyanate (Sigma Aldrich). Fluorescence was measured by laser scanning.

Statistical analysis. Specified analyses were performed using SigmaPlot version 11 software (Systat Software, Inc., San Jose, CA).

\section{ACKNOWLEDGMENTS}

This work was funded by a grant to SGUL by the Bill \& Melinda Gates Foundation and the Wellcome Trust, under the Grand Challenges in Global Health Initiative and by a grant to Harvard Medical School by the Bill \& Melinda Gates Foundation's Collaboration for AIDS Vaccine
Discovery / Comprehensive Antibody - Vaccine Immune Monitoring Consortium, grant number 38619. We thank Professors Ralf Wagner and Hans Wolf, University of Regensburg and GENEART AG for the CN54expressing plasmid and Dr Edward Tarelli, Medical Biomics Centre, St George's University of London for mass spectrometry analysis of the recombinant protein. $\mathrm{MC}$ is supported by the Sir Joseph Hotung Trust. We thank Sueli Vieira and Saba Hussein for work on gp140 cloning and expression.

\section{DISCLOSURE}

The authors declare no conflict of interest.

C 2010 Society for Mucosal Immunology

\section{REFERENCES}

1. Report on the global HIV/AIDS epidemic 2008 UNAIDS/08.25E/JC1510E (www.unaids.org).

2. Shattock, R.J. \& Moore, J.P. Inhibiting sexual transmission of HIV-1 infection. Nat. Rev. Microbiol. 1, 25-34 (2003).

3. Miller, C.J. et al. Propagation and dissemination of infection after vaginal transmission of simian immunodeficiency virus. J. Virol. 79, 9217-9227 (2005).

4. Mascola, J.R. et al. Protection of macaques against vaginal transmission of a pathogenic HIV-1/SIV chimeric virus by passive infusion of neutralizing antibodies. Nat. Med. 6, 207-10 (2000).

5. Parren, P.W. et al. Antibody protects macaques against vaginal challenge with a pathogenic $R 5$ simian/human immunodeficiency virus at serum levels giving complete neutralization in vitro. J. Virol. 75, 8340-8347 (2001).

6. Kozlowski, P.A. et al. Differential induction of mucosal and systemic antibody responses in women after nasal, rectal, or vaginal immunization: influence of the menstrual cycle. J. Immunol. 169, 566-574 (2002).

7. Mazzoli, S. et al. HIV-specific mucosal and cellular immunity in HIVseronegative partners of HIV-seropositive individuals. Nat. Med. $\mathbf{3}$, 1250-1257 (1997)

8. Devito, C. et al. Mucosal and plasma lgA from HIV-exposed seronegative individuals neutralize a primary HIV-1 isolate. AIDS 14, 1917-1920 (2000).

9. Ghys, P.D. et al. Cervicovaginal anti-HIV antibodies in HIV-seronegative female sex workers in Abidjan, Côte d'Ivoire. AIDS 14, 2603-2608 (2000).

10. Bélec, L. et al. Cervicovaginal secretory antibodies to human immunodeficiency virus type 1 (HIV-1) that block viral transcytosis through tight epithelial barriers in highly exposed HIV-1-seronegative African women. J. Infect. Dis. 184, 1412-1422 (2001).

11. Ogra, P. \& Ogra, S.S. Local antibody response to poliovirus in the human female genital tract. J. Immunol. 110, 1307-1311 (1973).

12. Wassen, L., Schön, K., Holmgren, J., Jertborn, M. \& Lycke, N. Local intravaginal vaccination of the female genital tract. Scand. J. Immunol. 44, 408-414 (1996).

13. Kozlowski, P.A., Cu-Uvin, S., Neutra, M.R. \& Flanigan, T.P. Comparison of the oral, rectal, and vaginal immunization routes for induction of antibodies in rectal and genital secretions of women. Infect. Immunol. 65, 1387-1394 (1997).

14. Curran, R.M. et al. Vaginal delivery of the recombinant HIV-1 clade-C trimeric gp140 envelope protein ZM96gp140 within novel rheologically structured vehicles elicits specific immune responses. Vaccine In press.

15. Schreckenberger, C. et al. Induction of an HPV 6bL1-specific mucosal IgA response by DNA immunization. Vaccine 19, 227-233 (2001).

16. Tommaso, A.D. et al. Induction of antigen-specific antibodies in vaginal secretions by using a nontoxic mutant of heat-labile enterotoxin as a mucosal adjuvant. Infect. Immun. 64, 974-979 (1996).

17. Walker, R.I. New strategies for using mucosal vaccination to achieve more effective immunization. Vaccine 12, 387-400 (1994).

18. Mestecky, J., Moldoveanu, Z. \& Russell, M.W. Immunologic uniqueness of the genital tract: challenge for vaccine development. Am. J. Reprod. Immunol. 53, 208-214 (2005).

19. Crowley-Nowick, P.A. et al. Normal uterine cervix: characterization of isolated lymphocyte phenotypes and immunoglobulin secretion. Am. J. Reprod. Immunol. 34, 241-247 (1995).

20. Belec, L., Tevi-Benissan, C., Lu, X.S., Prazuck, T. \& Pillot, J. Local synthesis of IgG antibodies to HIV within the female and male genital tracts during asymptomatic and pre-AIDS stages of HIV infection. AIDS Res. Hum. Retroviruses 11, 719-729 (1995). 


\section{ARTICLES}

21. Burton, D.R. et al. HIV vaccine design and the neutralizing antibody problem. Nat. Immunol. 5, 233-236 (2004).

22. Reitter, J.N., Means, R.E. \& Desrosiers, R.C. A role for carbohydrates in immune evasion in AIDS. Nat. Med. 4, 679-684 (1998).

23. McCaffrey, R.A., Saunders, C., Hensel, M. \& Stamatatos, L. N-linked glycosylation of the $\mathrm{V} 3$ loop and the immunologically silent face of gp120 protects human immunodeficiency virus type 1 SF162 from neutralization by anti-gp120 and anti-gp41 antibodies. J. Virol. 78, 3279-3295 (2004).

24. Pinter, A., Honnen, W.J., He, Y., Gorny, M.K. Zolla-Pazner, S. \& Kayman, S.C. The V1N2 domain of gp120 is a global regulator of the sensitivity of primary human immunodeficiency virus type 1 isolates to neutralization by antibodies commonly induced upon infection. J. Virol. 78, 5205-5215 (2004).

25. Kwong, P.D. et al. HIV-1 evades antibody-mediated neutralization through conformational masking of receptor-binding sites. Nature 420, 678-682 (2002).

26. Pantophlet, R., Wang, M., Aguilar-Sino, R.O. \& Burton, D.R. The human immunodeficiency virus type 1 envelope spike of primary viruses can suppress antibody access to variable regions. J. Virol. 83, 1649-1659 (2009).

27. Gnann, J.W., Nelson, J.A. \& Oldstone, M.B. Fine mapping of an immunodominant domain in the transmembrane glycoprotein of human immunodeficiency virus. J. Virol. 61, 2639-2641 (1987).

28. Moore, P.L. et al. Nature of nonfunctional envelope proteins on the surface of human immunodeficiency virus type 1. J. Virol. 80, 2515-2528 (2006).
29. Crooks, E.T. et al. A comparative immunogenicity study of HIV-1 virus-like particles bearing various forms of envelope proteins, particles bearing no envelope and soluble monomeric gp120. Virology 366, 245-262 (2007).

30. Su, L. et al. Characterization of a virtually full-length human immunodeficiency virus type 1 genome of a prevalent intersubtype $\left(\mathrm{C} / \mathrm{B}^{\prime}\right)$ recombinant strain in china. J Virol. 74, 11367-11376 (2000).

31. Rodenburg, C.M. et al. Near full-length clones and reference sequences for subtype $\mathrm{C}$ isolates of $\mathrm{HIV}$ type 1 from three different continents. AIDS Res. Hum. Retroviruses 17, 161-168 (2001).

32. Eckstein, P., Jackson, M.C., Millman, N. \& Sobrero, A.J. Comparison of vaginal tolerance tests of spermicidal preparations in rabbits and monkeys. J. Reprod. Fertil. 20, 85-93 (1969).

33. Li, M. et al. Human immunodeficiency virus type 1 env clones from acute and early subtype $B$ infections for standardized assessments of vaccineelicited neutralizing antibodies. J. Virol. 79, 10108-10125 (2005).

34. Buchacher, A. et al. Generation of human monoclonal antibodies against HIV-1 proteins; electrofusion and Epstein-Barr virus transformation for peripheral blood lymphocyte immortalization. AIDS Res. Hum. Retroviruses 10, 359-369 (1994).

35. HIV Molecular Immunology 2006/2007 LA-UR 07-4752 (Korber, B.T.M., Brander, C., Haynes, B.F., Koup, R., Moore, J.P., Walker, B.D. \& Watkins, D.I., eds) (Los Alamos National Laboratory, Theoretical Biology and Biophysics, Los Alamos, New Mexico. 